\title{
Effects of Van der Waals Bonding on the Compressive Mechanical Behavior of Bulk $\mathrm{Bi}_{2} \mathrm{Te}_{3}$ : A Molecular Dynamics Study
}

\author{
BEN HUANG, ${ }^{1}$ XUQIU YANG,${ }^{1,3}$ LISHENG LIU, ${ }^{1}$ \\ and PENGCHENG ZHAI ${ }^{1,2}$ \\ 1.-Department of Engineering Structure and Mechanics, Wuhan University of Technology, \\ Wuhan 430070, China. 2.-State Key Laboratory of Advanced Technology for Materials Synthesis and \\ Processing, Wuhan University of Technology, Wuhan 430070, China. 3.—e-mail: yangxuqiu@163.com
}

Along the $c$ axis of the crystal lattice, $\mathrm{Bi}_{2} \mathrm{Te}_{3}$ has periodic quintuple layers “-Te1-Bi-Te2-Bi-Te1-" which are connected by Van der Waals bonding. The weak bonding between Te1-Te1 layers substantially affects the mechanical properties of $\mathrm{Bi}_{2} \mathrm{Te}_{3}$. In the work discussed in this paper, the molecular dynamics method was used to study the mechanical properties of cuboid single-crystal of bulk $\mathrm{Bi}_{2} \mathrm{Te}_{3}$ under compressive loads. The emphasis was on the effects of the Van der Waals bonding on the deformation and failure mechanism. The molecular dynamics simulation results revealed that Van der Waals bonding has a dominant effect on the mechanism of deformation, and fundamentally determines the ultimate stress and fracture strain. Furthermore, the compressive load along the $a$ and $c$ axes lead to quite different failure behavior, which can be distinguished by their specific effects on the deformation of the Van der Waals bonding. However, only models with the load along the $a$ axis dramatically demonstrate the effect of strain rate on the stress-strain curves, in accordance with the poor structural stability.

Key words: $\mathrm{Bi}_{2} \mathrm{Te}_{3}$, Van der Waals bonding, compression properties, molecular dynamics

\section{INTRODUCTION}

$\mathrm{Bi}_{2} \mathrm{Te}_{3}$, the first efficient thermoelectric material to be discovered, is widely used in commercial applications. In recent years, $\mathrm{Bi}-\mathrm{Te}$ alloys with better thermoelectric performance have frequently been reported. Because materials with designed structures can have desirable characteristics, optimization of materials is regarded as an important means of improving the thermoelectric properties of $\mathrm{Bi}_{2} \mathrm{Te}_{3}$. As a result, nanomaterials and nanocomposites are attracting much attention. Much research has shown that the $Z T$ of thermoelectric materials can be substantially improved by use of small-dimension

(Received July 14, 2014; accepted October 31, 2014; published online December 2, 2014) materials. ${ }^{1,2}$ Specifically, thermal conductivity, $\kappa$, is reduced by phonon scattering, ${ }^{3,4}$ and the power factor, $\alpha^{2} \sigma$, is increased by quantum confinement effects. ${ }^{5}$ For instance, in 2001, Venkatasubramanian reported a $\mathrm{Bi}_{2} \mathrm{Te}_{3} / \mathrm{Sb}_{2} \mathrm{Te}_{3}$ superlattice thin film with a $Z T$ of $2.4{ }^{6}$ For the purpose of large-scale commercial application, nanocomposite materials seem quite promising, and their preparation is comparatively simple. In 2007, Tang prepared $\mathrm{Bi}_{2} \mathrm{Te}_{3}$ bulk materials with a layered nanostructure by combining meltspinning with spark plasma sintering. ${ }^{7}$ The peak $Z T$ value of 1.35 was obtained at $300 \mathrm{~K}$, because the layered nanostructure resulted in optimized transport properties of the carriers and phonons. It is also worth noting that, for better application of $\mathrm{Bi}_{2} \mathrm{Te}_{3}$, the mechanical stability of the designed structure is also of vital importance. Despite this, relatively few studies have been conducted on the mechanical properties of $\mathrm{Bi}_{2} \mathrm{Te}_{3}$. 
The crystal structure of $\mathrm{Bi}_{2} \mathrm{Te}_{3}$ is rhombohedral, with space group $R \overline{3} \mathrm{~m}$, containing five atoms in one unit cell, which can, for convenience, be regarded as a hexagonal unit cell. ${ }^{8}$ In this hexagonal unit cell, $a=4.47 \AA$ and $c=30.49 \AA$. As shown in Fig. 1 , along the $c$ axis, $\mathrm{Bi}_{2} \mathrm{Te}_{3}$ has periodic quintuple layers "-Te1-Bi-Te2-Bi-Te1-" which are connected by Van der Waals bonding. Compared with the covalent and ionic bonding in the crystal lattice, ${ }^{9}$ this weak bonding between the Te1-Te1 layers substantially limits the mechanical properties of $\mathrm{Bi}_{2} \mathrm{Te}_{3}$. This should, therefore, be a noteworthy structural feature for investigation of the mechanism of deformation of the thermoelectric material.

As a reliable theoretical method in various fields, molecular dynamics (MD) simulation is quite useful in structure analysis at the atomic level. In 2008, Agrawal reported MD simulation of the variation of the six carbon-carbon bond lengths and the bond angles of the hexagons in single-walled carbon nanotubes as a function of tensile strain. ${ }^{10}$ In 2012 , to provide better simulating systems to allow for chemical reactions, Svaneborg extended the largescale atomic/molecular massively parallel simulator (LAMMPS) $^{11}$ to support directional bonds and dynamic bonding. He gave a coarse-grained deoxyribose nucleic acid (DNA) model as an illustrative example. ${ }^{12}$

The MD method is gradually being applied to studies of thermoelectric materials also. In 2008, Huang studied the bond structure of $\mathrm{Bi}_{2} \mathrm{Te}_{3}$ by use of density functional theory (DFT) calculations, and, on the basis of the results, was the first to develop a

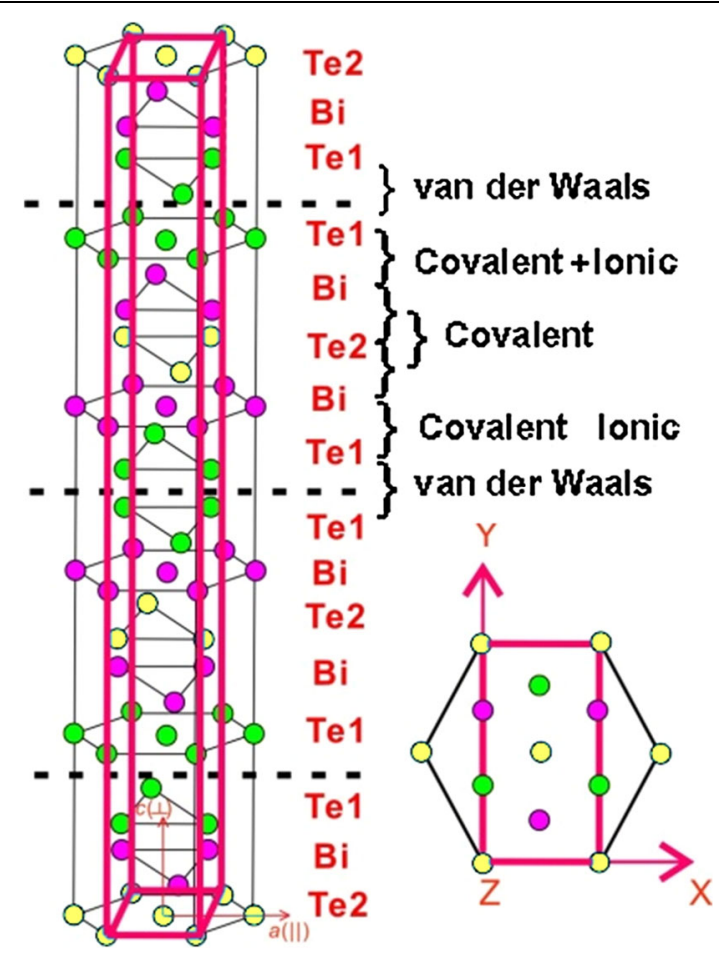

Fig. 1. The crystal structure of $\mathrm{Bi}_{2} \mathrm{Te}_{3}$. classical interatomic potential for MD. Phonon and electron transport in $\mathrm{Bi}_{2} \mathrm{Te}_{3}$ were also investigated, by use of the multiscale approach. ${ }^{13}$ Two years later, Tong investigated intact $\mathrm{Bi}_{2} \mathrm{Te}_{3}$ bulk with this potential. $^{9}$ In his MD study, calculated lattice constants, linear thermal expansion coefficients, and independent elastic constants were in good agreement with previous experimental and theoretical results, confirming the feasibility of potential selection for mechanics research. Furthermore, considering the mechanical properties of nanostructures, the failure behavior of $\mathrm{Bi}_{2} \mathrm{Te}_{3}$ nanowires under $a$ and $c$ axis tension have been studied by MD and the effect of strain rate was also taken into account. ${ }^{14}$

In the work discussed in this paper, the effects of the Van der Waals bonding on the compressive mechanical behavior of single-crystal bulk $\mathrm{Bi}_{2} \mathrm{Te}_{3}$ were investigated by $\mathrm{MD}$ simulation. The results might aid the preparation of high-efficiency $\mathrm{Bi}_{2} \mathrm{Te}_{3}$ materials with stable structures.

\section{SIMULATION METHOD AND SETUP}

For the MD study of the role of Van der Waals bonding, the LAMMPS software package (Sandia National Laboratories, Livermore, CA, USA) ${ }^{11}$ was used as solver and analyzer. Given that the interatomic potential is the core of any MD simulation, Huang's potential ${ }^{13}$ was adopted because of its rationality. 9,14

The Cartesian reference axis of the simulation cell shown in Fig. 1 is the same as in Ref. 15, which means the $x$ and $z$ axes represent the $a$ and $c$ principal axes, respectively in the hexagonal system also. For the anisotropy of single-crystal bulk $\mathrm{Bi}_{2} \mathrm{Te}_{3}$, two models were established to simulate the $x$ and $z$ axes uniaxial compression tests. The sizes of the simulated cuboid supercells were $273.312 \AA \times$ $92.043 \AA \times 91.464 \AA$ and $91.104 \AA \times 92.043 \AA \times$ $274.392 \AA$, with the same total atom number of 68,040 .

The settings of the two models are the same as in LAMMPS except for the loading direction. Periodic boundary conditions were applied in all the directions of the supercells. The Maxwell-Boltzmann distribution was used to assign the initial atomic velocities according to the given temperature of $300 \mathrm{~K}$. The bulk $\mathrm{Bi}_{2} \mathrm{Te}_{3}$ was then thermally relaxed with a isothermal-isobaric (NPT) ensemble running 70,000 steps with a time step of $1 \mathrm{fs}$ to reach an equilibrium state. The temperature and the pressure were kept constant by the Nose-Hoover method. After full relaxation, the compressive loading process is realized by changing the size of the supercell along the $x$ or $z$ axis direction of the system with an canonical (NVT) ensemble and a strain rate of $5 \times 10^{8} \mathrm{~s}^{-1}$. The stepwise compressive strain is 0.002 , and each step is relaxed for some time. The compression-relaxation step is repeated until breakage of the single-crystal bulk $\mathrm{Bi}_{2} \mathrm{Te}_{3}$ 
occurs. For better observation, the atomic configuration is shown through the visual molecular dynamics (VMD) atomistic configuration viewer. ${ }^{16}$

\section{RESULTS AND DISCUSSION}

Theoretically, single-crystal material can be highly anisotropic, and, considering the layer structure of $\mathrm{Bi}_{2} \mathrm{Te}_{3}$, this phenomenon should be more distinct for properties along the $a$ and $c$ axes of the lattice; this is confirmed directly by the stressstrain curves for the $x$ and $z$ axes uniaxial

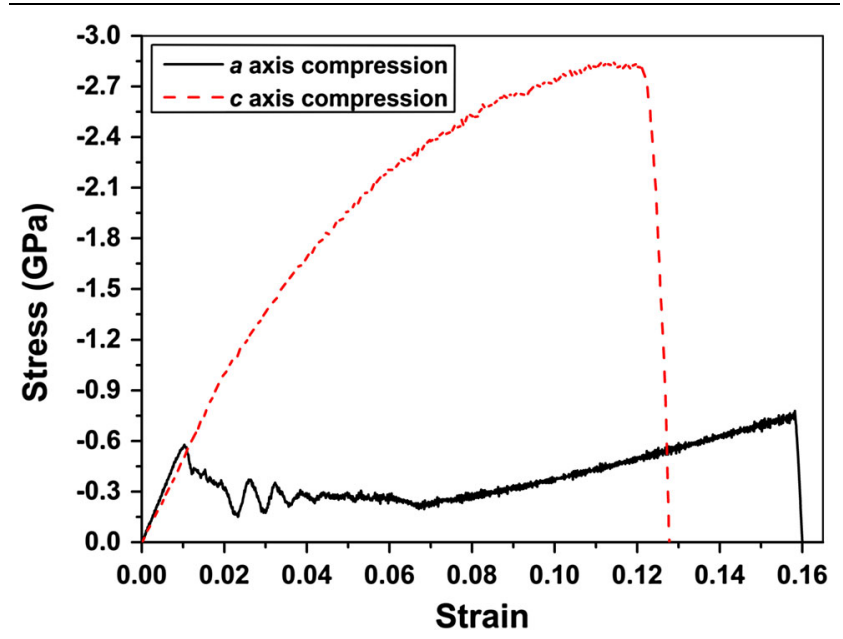

Fig. 2. Stress-strain curves for $a$ and $c$ axis uniaxial compression of single-crystal bulk $\mathrm{Bi}_{2} \mathrm{Te}_{3}$. compression MD simulation. As depicted in Fig. 2, these two curves are quite different. The elastic modulus along the $a$ axis is $58.6 \mathrm{GPa}$ whereas it is $48.8 \mathrm{GPa}$ along the $c$ axis. The calculated modulus of the bulk almost approaches those previously reported by Huang $(65.4 \mathrm{GPa}$ and $54.8 \mathrm{GPa}$ at $300 \mathrm{~K})^{13}$ and Jenkins $(68.47 \mathrm{GPa}$ and $47.68 \mathrm{GPa}$ at $280 \mathrm{~K}){ }^{15}$ The difference is more obvious from the structural strengths, with ultimate stresses of 0.75 $\mathrm{GPa}$ and $2.85 \mathrm{GPa}$ for the two models, and from the fracture strains of $16.0 \%$ and $12.8 \%$, respectively.

Furthermore, the bulk $\mathrm{Bi}_{2} \mathrm{Te}_{3}$ has quite unique failure behavior when compressive load is applied along the $a$ and $c$ axes. For $c$ axis compression, it can be described as regular brittle fracture, which is similar to the results from Tong's tension simulation of the nanowire. ${ }^{14}$ As the compressive deformation increases, the stress of the bulk structure grows almost linearly. Finally, the stress reaches the ultimate bearing capacity of the Van der Waals bonding between adjacent Te1 layers, which is much less than that of the covalent bonds. Cleavage fracture then occurs suddenly between the weakly bonded Te1 layers, as shown in Fig. 3a. Please note that in all the figures depicting atomic configurations in this paper, Te1 atoms are colored red whereas the other atoms ( $\mathrm{Bi}$ and $\mathrm{Te} 2$ ) are colored green for ease of recognition. Thus the Van der Waals bonding is between the red atoms belonging to the adjacent layers, which can also be observed as red bonds in the schematic diagram of the bonding. (Covalent bonds are shown in green.)

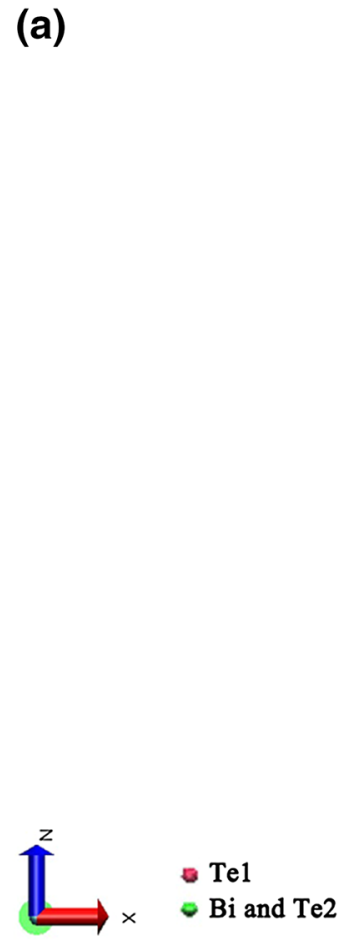

Fig. 3. The atomic configuration during failure for (a) $c$ axis compression and (b) a axis compression. (b)

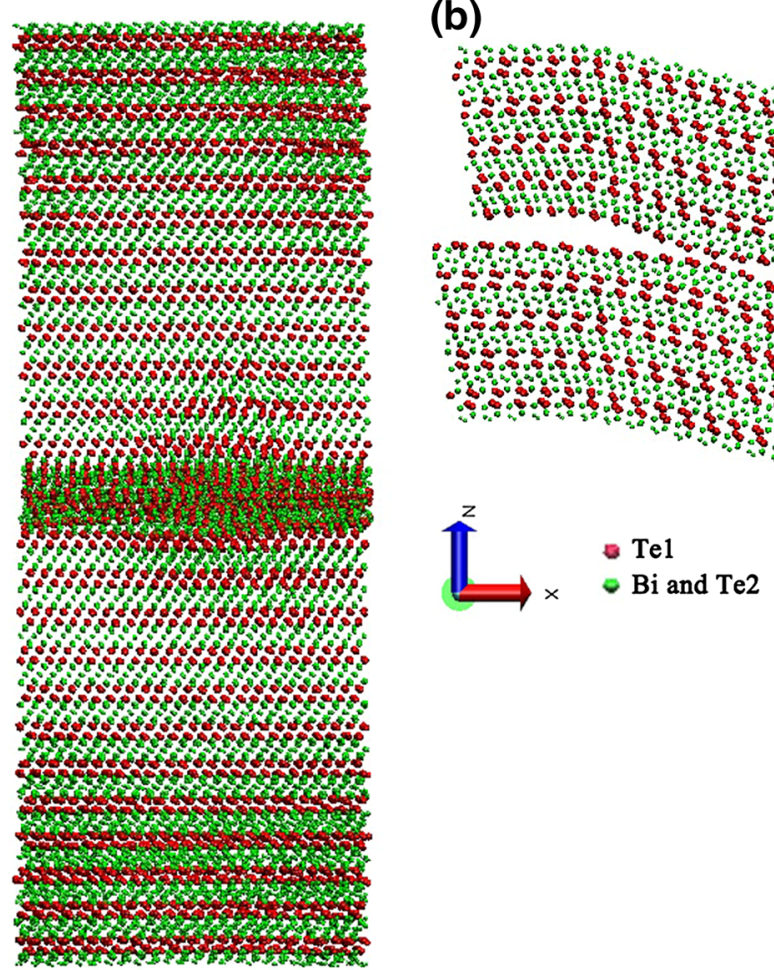




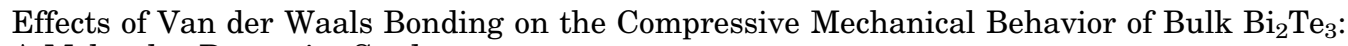
A Molecular Dynamics Study

(a1)

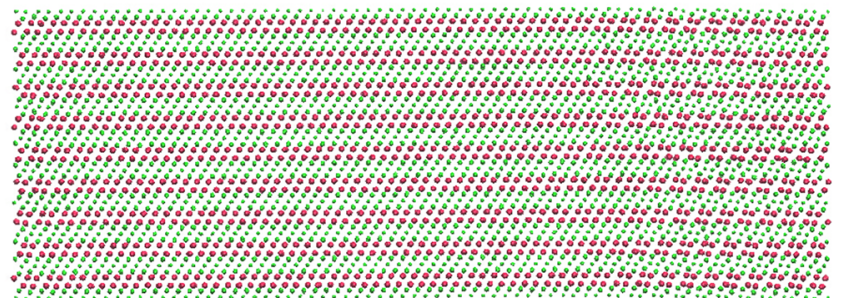
$\stackrel{z}{\longrightarrow} \begin{aligned} & \quad \mathrm{Te} 1 \\ & \mathrm{Xi}_{\mathrm{Bi}} \text { and } \mathrm{Te} 2\end{aligned}$

(b1)

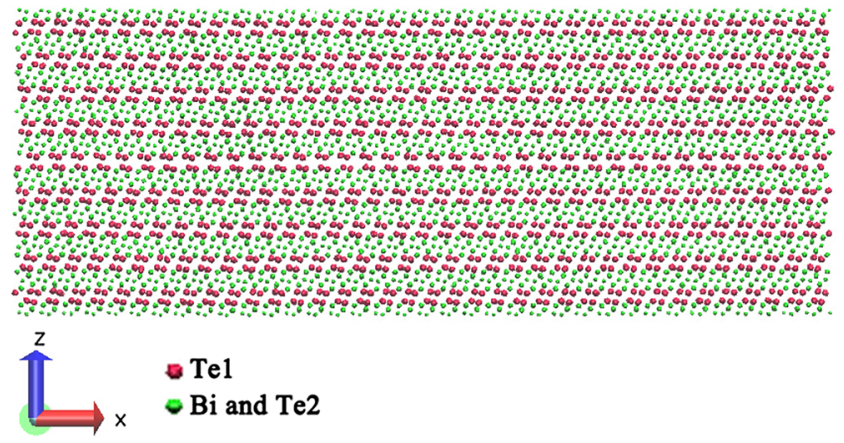

\section{(c1)}
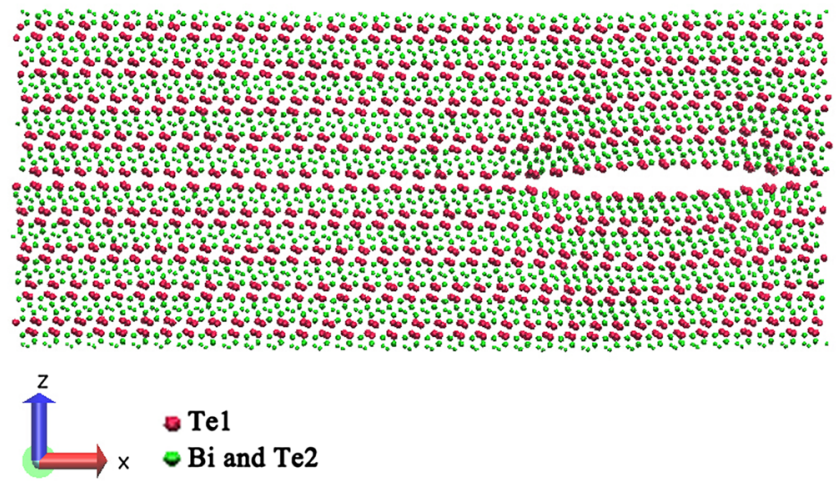

(a2)
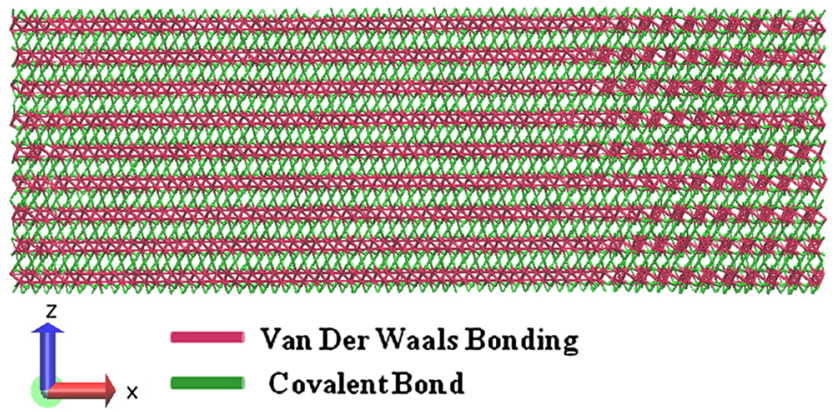

(b2)

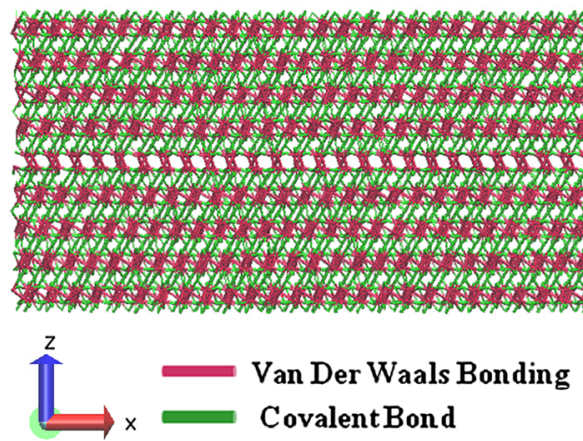

(c2)

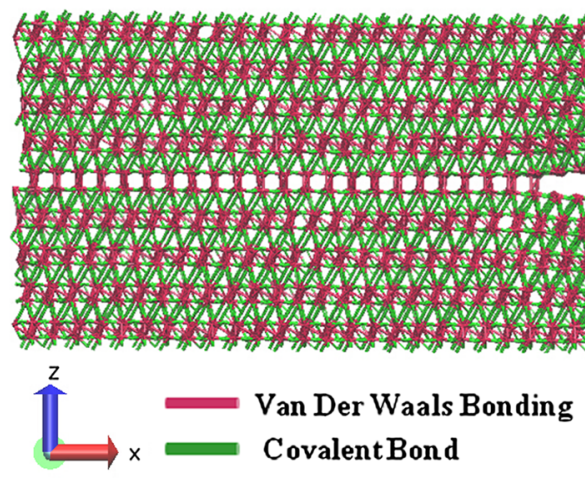

Fig. 4. Schematic diagram of atomic configuration and bonding for a axis compression at strains of (a) $\sim 1 \%$ (b) $7 \%$, and (c) $\sim 16 \%$.

Nevertheless, the process of deformation and failure for $a$ axis compression is very complex compared with the corresponding tension simulation. ${ }^{14}$ There is a peak in this curve at approximately $1 \%$ strain, which is quite irregular for compression of a brittle material. The atomic configuration gives some hints, indicating that parts of the Te1 layers bend slightly with $a$ axis pressure, leading to disorder of the atoms (as shown in Fig. 4a1). And, of course, some pairs of Te1 atoms become separated from each other, even beyond the range of the Van der Waals force, causing irreversible deformation of the structure (as shown in Fig. 4a2). Meanwhile, regional bending of the layer structure may induce stress release, as shown by the peak already mentioned, and the transient instability of the local structure will be apparent as oscillation in the subsequent process. This computational finding may even explain the lattice curvature in polycrystalline $\mathrm{Bi}_{0.5} \mathrm{Sb}_{1.5} \mathrm{Te}_{3}$ alloys processed by hot forging. ${ }^{17}$ It is apparent from Fig. $4 \mathrm{~b}$ that the structural disorder of the atoms gradually leads to an increase of the distance between specific adjacent Te1 layers, with formation of weak interfaces. Hereafter, with increasing pressure, the overall structure begins to support loads again at approximately $7 \%$ strain, so the stress-strain curve increases linearly. The elastic modulus here is only $4.9 \mathrm{GPa}$, much smaller than the value before the $1 \%$ strain, which also proves some form of irreversible deformation is happening during regional layer bending. At the same time, further disorder of the atoms is being caused by the increasing strain, eventually leading to separation of the weak Te1-Te1 interfaces at 

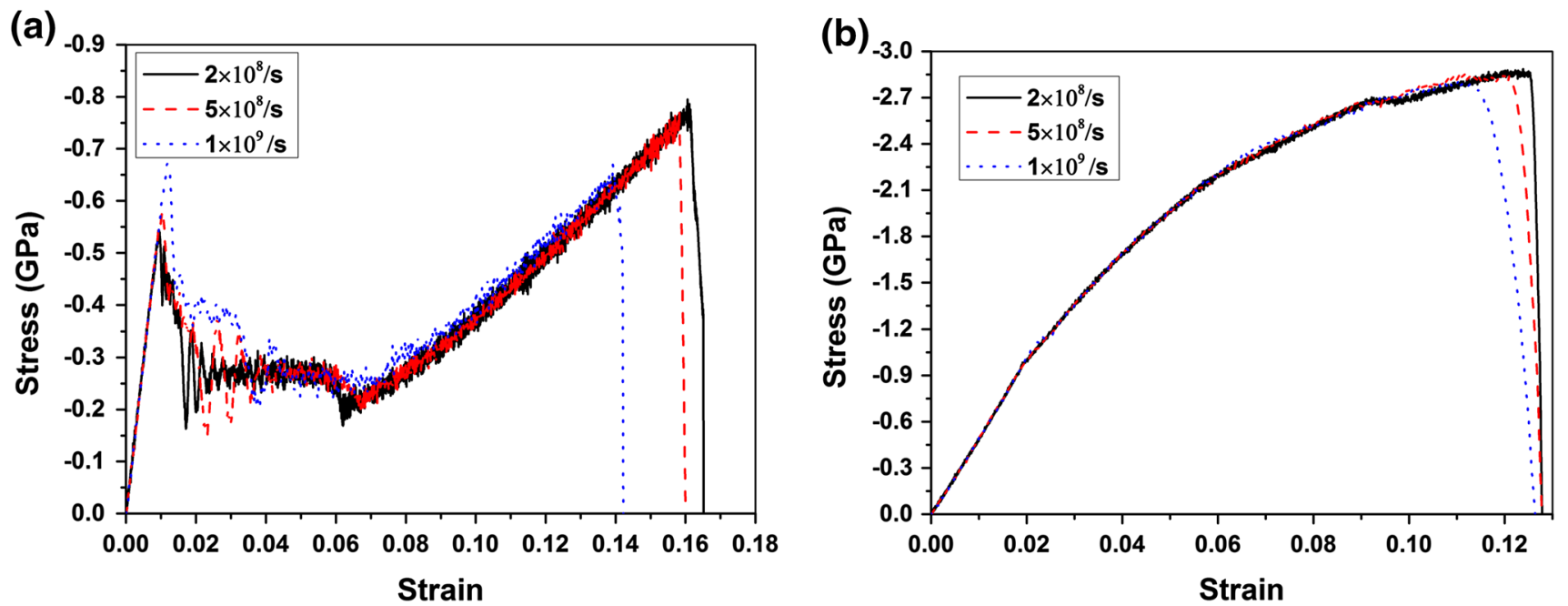

Fig. 5. The stress-strain curves of (a) a axis compression and (b) $c$ axis compression, with strain rates of $2 \times 10^{8} \mathrm{~s}^{-1}, 5 \times 10^{8} \mathrm{~s}^{-1}$ and $1 \times 10^{9} \mathrm{~s}^{-1}$.

approximately $16 \%$ strain (as shown in Fig. 4c). Soon after this the overall structure becomes disorganized, and the separated parts of the bulk undergo buckling failure with increasing load (as shown in Fig. 3b).

Because we know that buckling failure is related to loading rate, $a$ axis compression simulations were conducted with strain rates of $2 \times 10^{8} \mathrm{~s}^{-1}$, $5 \times 10^{8} \mathrm{~s}^{-1}$, and $1 \times 10^{9} \mathrm{~s}^{-1}$. The other settings in LAMMPS were kept the same as before. The strain rate effect is confirmed directly by the stress-strain curves shown in Fig. 5a. When the strain rate increases from $2 \times 10^{8} \mathrm{~s}^{-1}$ to $1 \times 10^{9} \mathrm{~s}^{-1}$, only the ultimate stress at the highest rate decreases to 0.67 $\mathrm{GPa}$; for the other two curves the value is almost constant at $0.75 \mathrm{GPa}$. Meanwhile, the fracture strain decreases from $16.5 \%$ to $14.2 \%$, a reduction of $13.94 \%$. And, obviously, the reduction in strength is not in equal proportions, in accordance with the unstable structure caused by the disordered atoms and the separated layers. By contrast, $c$ axis compression seems independent of loading rate, for the three curves coincide well in Fig. $5 \mathrm{~b}$. In fact, the $c$ axis compression is related to deformation or strain only, which means that if the distance between Te1Te1 layers reaches a critical value, this will result in internal stress greater than the strength of Van der Waals bonding, and the overall structure will fracture rapidly.

\section{CONCLUSIONS}

In this work, the MD method was used to investigate the effect of the Van der Waals bonding on the compressive mechanical behavior of single-crystal bulk $\mathrm{Bi}_{2} \mathrm{Te}_{3}$. The simulations indicate the compression properties of $\mathrm{Bi}_{2} \mathrm{Te}_{3}$ are highly anisotropic. Van der Waals bonding has a dominant effect on the mechanism of deformation, and fundamentally determines the ultimate stress and fracture strain.
Unique failure behavior can be observed in different directions: regular brittle fracture occurs on $c$ axis compression whereas buckling failure occurs on $a$ axis compression. Compression of the $a$ axis dramatically demonstrates the strain rate effect, because of its poor structural stability caused by disordered atoms and separated layers. However, $c$ axis compression leads to deformation only, solely for the interatomic distance affected by the loads. These results may be helpful for optimization of $\mathrm{Bi}_{2} \mathrm{Te}_{3}$ to achieve high $Z T$ and good mechanical stability. Much work on the mechanical mechanism must still be conducted, for example DFT study.

\section{ACKNOWLEDGEMENTS}

This work was financially supported by the National Program on Key Basic Research Project (973 Program, no. 2013CB632505), the National High-tech R\&D Program of China (863 Program, no. 2012AA051104), the International Science and Technology Cooperation Program of China (no. 2011DFB60150), and the National Natural Science Foundation of China (no. 10672127).

\section{REFERENCES}

1. L.D. Hicks, T.C. Harman, X. Sun, and M.S. Dresselhaus, Phys. Rev. B 53, R10493 (1996).

2. X. Sun, Z. Zhang, and M.S. Dresselhaus, Appl. Phys. Lett. 74, 4005 (1999).

3. R.G. Yang, G. Chen, and M.S. Dresselhaus, Phys. Rev. B 72, 125418 (2005).

4. W. Kim, J. Zide, A. Gossard, D. Klenov, S. Stemmer, A. Shakouri, and A. Majumdar, Phys. Rev. Lett. 96, 045901 (2006).

5. D.A. Broido and N. Mingo, Phys. Rev. B 74, 195325 (2006).

6. R. Venkatasubramanian, E. Siivola, T. Colpitts, and B. O'Quinn, Nature 413, 597 (2001).

7. X.F. Tang, W.J. Xie, H. Li, W.Y. Zhao, Q.J. Zhang, and M. Niino, Appl. Phys. Lett. 90, 012102 (2007).

8. J.R. Wiese and L. Muldawer, J. Phys. Chem. Solids 15, 13 (1960). 
Effects of Van der Waals Bonding on the Compressive Mechanical Behavior of Bulk $\mathrm{Bi}_{2} \mathrm{Te}_{3}$ :

A Molecular Dynamics Study

9. Y. Tong, F.J. Yi, L.S. Liu, and P.C. Zhai, Comp. Mater. Sci. 48,343 (2010).

10. P.M. Agrawal, B.S. Sudalayandi, L.M. Raff, and R. Komanduri, Comput. Mater. Sci. 41, 450 (2008).

11. S.J. Plimpton, J. Comp. Phys 117, 1 (1995).

12. Carsten Svaneborg, Comput. Phys. Commun. 183, 1793 (2012).

13. B.L. Huang and M. Kaviany, J. Phys. Rev. B 77, 125209 (2008).
14. Y. Tong, F.J. Yi, L.S. Liu, and Q.J. Zhang, J. Electron. Mater. 39, 1730 (2010).

15. J.O. Jenkins, J.A. Rayne, and R.W. Ure, Phys. Rev. B 5, 3171 (1972).

16. W. Humphrey, A. Dalke, and K. Schulten, J. Mol. Graph. 14, 33 (1996).

17. J.J. Shen, T.J. Zhu, X.B. Zhao, S.N. Zhang, S.H. Yang, and Z.Z. Yin, Energy Environ. Sci. 3, 1519 (2010). 